\title{
Starting with PD Enables Diversity of Later Treatments
}

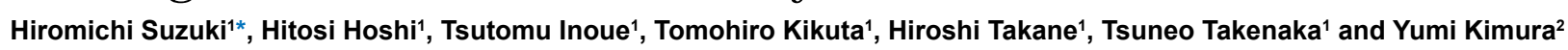

${ }^{1}$ Department of Nephrology, Saitama Medical University, Japan

${ }^{2}$ Nursing Department, Saitama Medical University Hospital, Japan

\begin{abstract}
Background: Various reasons for "Peritoneal Dialysis (PD) First" rather than hemodialysis (HD) have been presented, such as better preservation of residual renal function (RRF), longer survival, and lower incidence of hospitalization. In spite of these advantages for "PD First", in Japan as well as in the United States the annual rate of patients receiving PD has been reduced to less than $10 \%$. One of the major reasons against selecting PD is that a large proportion of PD patients are transferred from PD to HD in less than 5 years. Our "PD First" policy is based on the diversity of modalities available after discontinuing PD therapy. The purpose of this study was to examine the follow-up of patients who selected "PD First" as the initial treatment of end-stage renal disease (EDRD) between April 1997 and December 2010.
\end{abstract}

Methods: Sex, age, primary underlying diseases and selection of modalities were collected retrospectively.

Results: A total of 377 (59.2 \pm 8.3 years old; female/male: 255/122) patients were introduced to PD therapy as "PD First." Patients who were very old, with cardiovascular problems, senile dementia, and neoplasms that were forced to select PD were excluded. One hundred and sixty patients started HD as complementary dialysis therapy and then continued with PD + HD in combination until transfer to HD, transplantation or home HD. Among them, 10 patients received transplants and 22 patients were transferred to home HD. One hundred and twenty eight patients were switched from PD to HD for various reasons. Overall patients' survival after 5 and 10 years was $84.8 \%$ and $55.8 \%$, respectively.

Conclusion: Our data shows a diversity of modalities for selection after discontinuing PD therapy alone, as well as providing a rationale to support PD as the initial renal replacement modality for end-stage renal disease patients.

Keywords: Peritoneal dialysis; Hemodialysis; Combination therapy; Home hemodialysis; Renal transplantation

\section{Introduction}

The selection of a dialysis modality is important for patients who start Renal Replacement Therapy (RRT). However, more than $90 \%$ of patients submit to in-center Hemodialysis (HD) without receiving sufficient information. There are at least three modalities of RRT: transplantation, continuous ambulatory Peritoneal Dialysis (PD), and HD. These modalities differ clinically, and offer advantages and disadvantages for an individual patient. At present, of the triad of these modalities, transplantation would be the ideal. However, in Japan, several factors such as the shortage of donors, advanced age of patients, and increases in diabetic patients preclude transplantation as a readily available option for most patients [1]. Besides, a prospective randomized study comparing $\mathrm{HD}$ and $\mathrm{PD}$ outcomes are relatively rare in practice, because it is difficult to assign patients randomly to two types of dialysis therapy $[2,3]$.

Recently, Residual Renal Function (RRF) has been recognized as an important marker of outcome of patients on both PD and HD [4-6]. $\mathrm{RRF}$ is an especially powerful indicator for better removal of middle and larger molecular weight toxins and organic acids that accumulate during dialysis, and PD is proposed for initial dialysis therapy. Then, as RRF decreases a switch from PD to HD would be carried out.

There is the concern however, that in the absence of RRF only a fraction of PD patients are capable of clearing small molecules to acceptable levels without the use of automated PD [7].

Combination therapy with HD and PD allows for the partial separation of the two essential components of effective RRT, i.e., fluid removal and solute clearance [8-13]. In spite of using PD and HD in combination, peritoneal membranes gradually lose the ability to maintain adequate dialysis and many patients switch to HD only [14].
We have utilized the sequence of PD first, PD and HD in combination, and home HD (HHD) and renal transplantation at the Kidney Disease Center, Saitama Medical University Hospital. The serial data obtained from these patients are expected to provide some clues for selection and alternation of dialysis modalities. The aim of this study first examined the diversity of RRT when PD therapy was unable to maintain adequate solute and/or fluid removal. If this diversity prolonged patients' survival, proposal of "PD" first as an initial RRT will be validated.

\section{Patients and Methods}

This was a retrospective observational cohort study. Incident PD patients aged 18 years or older at Saitama Medical University Hospital between April 1997 and December 2010 were screened for study eligibility. All patients were regularly monitored at our center and had started their PD treatment at least 6 months before enrollment.

A total of 377 (59.2 \pm 8.3 years old; female/male: $255 / 122)$ patients were introduced to PD therapy as "PD First." All patients were followed till death or to the end of the study on December 31,2010. Demographic data, the cause of end-stage renal disease, and relevant biochemical

*Corresponding author: Hiromichi Suzuki, Department of Nephrology, Saitama Medical University, 38 Moroyama-machi, Iruma-gun, Saitama, 350-0495 Japan, Tel: +81-49276-1620; Fax: +81-49295-7338; E-mail: iromichi@saitama-med.ac.jp

Received December 27, 2013; Accepted January 22, 2014; Published January 26, 2014

Citation: Suzuki H, Hoshi H, Inoue T, Kikuta T, Takane H, et al. (2013) Starting with PD Enables Diversity of Later Treatments. J Nephrol Therapeutic S1: 004 doi:10.4172/2161-0959.S1-004

Copyright: ( 2013 Suzuki $\mathrm{H}$, et al. This is an open-access article distributed under the terms of the Creative Commons Attribution License, which permits unrestricted use, distribution, and reproduction in any medium, provided the original author and source are credited. 
data were collected. Transplantation and frequent HD were censored observations. The causes of death were categorized as cardiovascular, malignancy, and others. Patients on PD were permanently transferred to HD due to inadequate dialysis, peritonitis, ultrafiltration failure, exit-site infection, tunnel infection, and mechanical or operational problems.

Baseline data were collected regarding patients' demographic characteristics, laboratory values and duration on PD.

Informed consent was obtained at the start of PD therapy. This study was performed in accordance with the principles of the World Medical Association Declaration of Helsinki and was conducted in the Kidney Disease Center in Saitama Medical University Hospital, Saitama, Japan.

\section{Patient monitoring}

Patients were followed every month during the study period. At each clinic visit, serum creatinine, electrolyte concentrations, complete blood count, and other serum chemistries (uric acid, glucose, and liver enzymes) were measured. Indices of the adequacy of dialysis, including weekly CCr, were calculated using the Adequest computer program, version 2.0 (Baxter Healthcare, Tokyo, Japan) for Windows. Chest radiographs were obtained regularly, and cardiothoracic index was calculated according to established methods.

During the study, target home blood pressure (BP) was $130 / 80$ $\mathrm{mmHg}$ or lower and home BP measurements were encouraged. The selection of an antihypertensive agent depended on the physicians' preference. Subjects were treated with recombinant human erythropoietin (rHuEPO) as necessary and their hemoglobin levels were maintained between 10 to $11 \mathrm{~g} / \mathrm{dL}$. Subjects were given oral iron supplementation if they were diagnosed with iron deficiency.

Subjects with parathyroid hormone levels greater than $500 \mathrm{pg} / \mathrm{ml}$ were treated with $1,25(\mathrm{OH})_{2} \mathrm{D} 3$ and $\mathrm{CaCO}_{3}$ supplements, while patients with levels lower than $70 \mathrm{pg} / \mathrm{ml}$ were treated with $\mathrm{CaCO}_{3}$ to reduce the degree of hypophosphatemia. Doses were adjusted based on serum levels of calcium and phosphate. Lipid lowering drugs, primarily statin derivatives, were administered if serum cholesterol levels exceeded 240 $\mathrm{mg} / \mathrm{dl}$.

\section{Regular treatment modality in the kidney center in saitama medical university}

More than $60 \%$ of patients were treated with a standard PD regimen that consisted of three to four daily exchanges of 1.5 or 2 liters of dialysate, while other patients used one to two daily exchanges of dialysate. The strength of the bags was individualized to maintain the desired weight. Dwell times were also individualized to maximize overall ultrafiltration volumes. Mean daily dietary intake was recorded from individual 24-hour food records during a three-day period at the start of the study. All subjects consumed between 0.8 and $1.0 \mathrm{~g}$ of protein $/ \mathrm{kg} /$ day and their energy intake exceeded $25 \mathrm{kcal} / \mathrm{kg} /$ day and salt intake was restricted to less than $9 \mathrm{~g}$ daily throughout the study.

\section{Combination of PD and HD}

The criteria for introduction of HD as in combination therapy was determined as less than creatinine clearance of $45 \mathrm{~L} /$ week calculated by Adequest or as fluid overload. A $4 \mathrm{hr} \mathrm{HD}$ was added once a week after 6 consecutive days of PD. On the morning of HD, the PD dialysate was drained before HD. HD was carried out using a bicarbonate dialysate and a dialyzer with a polysulfone dialysis membrane.

\section{Statistical analysis}

Results are expressed as mean \pm standard error of the mean. Statistical analyses used the Student's $t$-test for unpaired samples and the Mann-Whitney test for comparison of means. Survival times were estimated from Kaplan-Meier curves. Statistical significance was set at $p<0.05$. The analyses were performed using JMP software, version 9 (JMP, A Business Unit of SAS, NC USA).

\section{Results}

\section{Clinical characteristics at the start of CAPD}

Table 1 shows the baseline characteristics at the start of PD for all patients. A total of 377 were analyzed in our study with an average age of 59.2 years (male, 67.6\%). Chronic glomerulonephritis, diabetes mellitus and hypertension accounted for the three most common causes of ESRD. Laboratory data including serum albumin, uric acid, creatinine, and hemoglobin are shown in Table 1.

\section{Outcome}

The mean follow-up period was $12.3 \pm 3.9$ years (range 3-16 years). As shown in Figure 1, patients who selected PD as the initial RRT, were diverted from various modalities of dialysis therapy.

In the Table 2, the following data is shown.

\section{Baseline characteristics of patients utilized PD and HD in combination}

The baseline characteristics of patients who utilized PD and HD in combination are shown. A total of 160 were analyzed in our study with an average age of 52.4 years (male, 67.5\%). Chronic glomerulonephritis,

\begin{tabular}{|c|c|}
\hline Total number & 377 \\
\hline Sex ratio (M/F) & $255 / 122$ \\
\hline Age (years) & $59.2 \pm 8.3$ \\
\hline Underlying diseases & 218 \\
\hline Glomerulonephritis & 100 \\
\hline Diabetes Mellitus & 28 \\
\hline Hypertension & 31 \\
\hline Others and Unknown & $37.2 \pm 5.7$ \\
\hline Albumin $(\mathrm{g} / \mathrm{L})(\mathrm{mmol} / \mathrm{L})$ & $839 \pm 256$ \\
\hline Creatinine $(\mu \mathrm{mol} / \mathrm{L})$ & $4.23 \pm 0.97$ \\
\hline Uric acid $(\mathrm{m} \mathrm{mol} / \mathrm{L})$ & $99.6 \pm 22.6$ \\
\hline Hemoglobin $(\mathrm{g} / \mathrm{L})$ & \\
\hline
\end{tabular}

Table 1: Characteristics of all subjects.

PD $\quad 377$

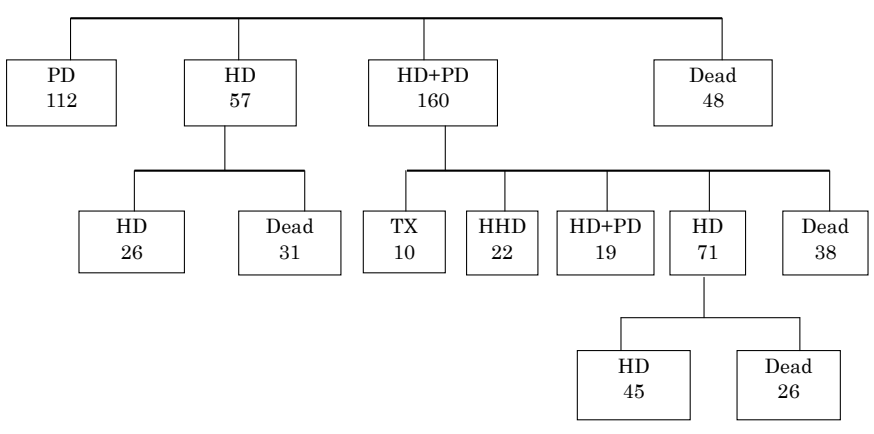

Figure 1: Follow up chart of all patients. 


\begin{tabular}{|c|c|c|c|c|c|}
\hline & HD and PD & PD and HD to HD & Directly to HD & HHD & Transplantation \\
\hline Total number & 160 & 71 & 57 & 10 & 22 \\
\hline Sex ratio $(M / F)$ & $108 / 52$ & $52 / 19$ & $41 / 16$ & $(2 / 8)$ & $(18 / 4)$ \\
\hline Age (years) & $52.4 \pm 9.5$ & $48.3 \pm 7.2$ & $65.1 \pm 8.9$ & $47.5 \pm 9.2$ & $46.8 \pm 11.5$ \\
\hline \multicolumn{6}{|l|}{ Underlying diseases } \\
\hline Glomerulonephritis & 87 & 29 & 30 & 7 & 19 \\
\hline Diabetes Mellitus & 51 & 30 & 15 & 1 & 0 \\
\hline Hypertension & 15 & 7 & 3 & 1 & 2 \\
\hline Others and Unknown & 7 & 5 & 9 & 0 & 1 \\
\hline Duration of PD (years) & $3.3 \pm 1.9$ & $3.7 \pm 2.1$ & $4.2 \pm 2.9$ & $6.8 \pm 2.5$ & $8.4 \pm 1.1$ \\
\hline
\end{tabular}

PD: peritoneal dialysis; HD: hemodialysis; HHD: home hemodialysis; M: male; F: female

Table 2: Characteristics of patients followed.

diabetes mellitus and hypertension accounted for the three most common causes of ESRD. Duration of PD was 3.3 years.

\section{Baseline characteristics of patients utilized PD and HD in combination transferred to HD}

The baseline characteristics of patients who utilized PD and HD in combination and were ultimately transferred to HD are shown. A total of 71 were analyzed in our study with an average age of 48.3 years (male, 74.2\%). Chronic glomerulonephritis and diabetes mellitus accounted for the two most common causes of ESRD. Duration of PD including combination therapy was 3.7 years.

\section{Baseline characteristics of patients on PD were directly transferred to HD}

The baseline characteristics of patients on PD who were directly transferred to HD are shown. A total of 57 were analyzed in our study with an average age of 64.1 years (male, 76.9\%). Chronic glomerulonephritis and diabetes mellitus accounted for the two most common causes of ESRD. Duration of PD was 4.2 years.

\section{Baseline characteristics of patients on PD were transferred to HHD or renal transplantation}

The baseline characteristics of patients on PD who were transferred to HHD or renal transplantation are shown. A total of 22 patients were transferred to HHD and 10 received renal transplants. Ages of these patients were 48.1 and 45.7 years old, respectively. Duration of PD was 6.8 and 8.4 years respectively. Chronic glomerulonephritis accounted for the most common causes of ESRD in both group.

\section{Patient survival}

A Kaplan-Meier curve of patients' survival is shown in Figure 2. Cumulative proportional patient survival at 3,5 and 10 years was $98.1 \%, 84.8 \%$ and $55.8 \%$.

\section{Discussion}

The clinical results from our observations suggest that the sequence of starting with PD, followed by the combination of PD + HD, and finally with HHD or renal transplantation is a useful and successful sequence of treating a subset of patients with ESRD. This approach of starting PD in patients that choose to do so, adding HD if indicated, and eventually switching to HHD or renal transplantation when combination therapy is no longer indicated, would fit well with the concept of case integration. Van Biesen et al. [15] showed that patients who were started on PD and then transferred to HD in an integrated approach had better outcomes than patients who were treated with HD alone.
Previously, we reported that the mean age of patients who selected "PD first" were younger compared with that of patients on HD [16]. In the present study, there were no differences in mean age of patients who needed HD as complementary dialysis modality and those patients who started PD as "PD first", indicating that a larger portion of PD patients needed HD as complementary dialysis modality as stated elsewhere [12]. Besides, in spite of treatment with PD and HD in combination, approximately $45 \%$ of patients left from PD therapy. This was probably due to younger and diabetic patients, because young subjects needed more dialysis and diabetic patients had various complications.

A PD-first recent analysis attempted to look at this model of integrated dialysis care by performing a retrospective analysis of patients who started on PD and then switched to HD, as compared with those patients who spent their entire ESRD course on HD policy may also have particular advantages for those likely to receive a kidney transplant within 2-3 years of initially dialysis. There is a strong likelihood that such patients will maintain RRT with its associated advantages until the transplant is performed. Most importantly, patients themselves should be intimately involved in the decision-making throughout their treatment period [17]. Our goal as nephrologists is to prolong life while maintaining QOL. In achieving this, patient preference is an important consideration. All subjects acknowledged that their quality of life had improved after they began combination therapy. Unfortunately, their quality of life before and after the study started was not evaluated. This subjective assessment of improved quality of life induces increases food

\section{Survival rate (\%)}

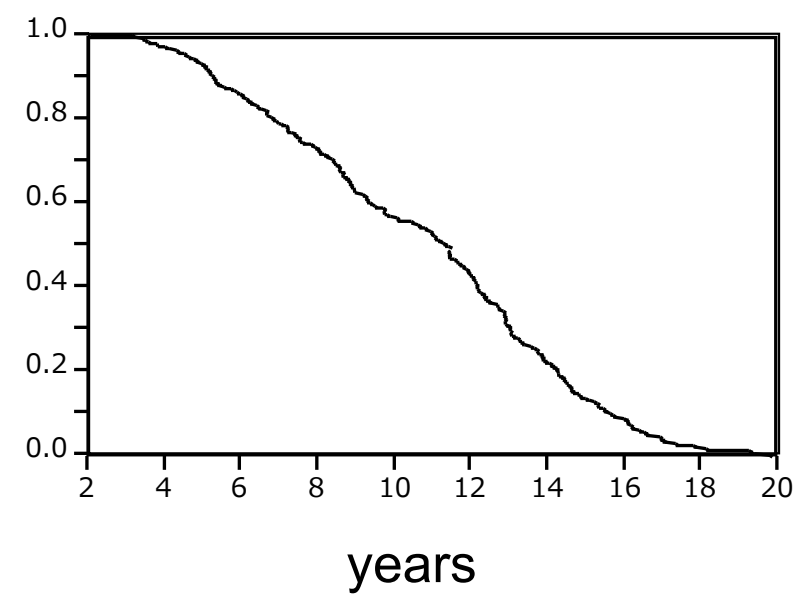

Figure 2: Kaplan-Meier survival curve. 
Citation: Suzuki H, Hoshi H, Inoue T, Kikuta T, Takane H, et al. (2013) Starting with PD Enables Diversity of Later Treatments. J Nephrol Therapeutic S1: 004 doi:10.4172/2161-0959.S1-004

Page 4 of 4

intake and may have contributed to the increase in serum albumin.

Patient survival of PD patients have been reported from Asian countries are greater than those in other countries. For example, in Japan, patient survival rates were $67.4 \%$ at 5 years and $48.6 \%$ at 10 years [18]. In Korea, 5- and 10- year patient survival rates were reported $69.8 \%$ and $51.8 \%$ respectively [19]. In our study, patients who selected as an initial therapy for RRT survived longer than those reported previously. The previous observational data discussing the superiority of survival rate between $\mathrm{HD}$ and $\mathrm{PD}$ without $\mathrm{HD}$ complementation failed to conclusion, because of complicated obstacles such as baseline data [2]. From our present study combining with the previous data, "PD" first might be one of the selections for RRT at least in Asian countries.

Our study is limited by its observational nature. We were unable to determine any absolute contraindications to home dialysis that were otherwise unknown. We also did not study the perception and availability of caregivers. Furthermore, this study was performed in a center that favored a "home dialysis first" approach. Our home dialysis prevalence statistics showing PD of $25 \%$ and HHD of $6 \%$ is relatively higher than current Japanese statistics [1].

Finally, this study is not a multicenter study, and a center-specific effect may exist. Furthermore, we are not suggesting that all patients with ESRD should be started on PD rather than HD. There are a significant number of patients for whom PD is not suitable for medical reasons. There are others who, for reasons of their own, are simply not willing to take on a home-based modality. Moreover, in this study, the causes of withdrawal from PD therapy were not analyzed. Our results will help to understand the origin of patients choosing a dialysis modality and reinforces the complementary nature of these dialysis modalities.

In conclusion, the clinical results from our observations suggest that PD first, followed by combined mode therapy, is a useful and successful sequence of treating a subset of patients with ESRD.

\section{Disclosures}

The authors declare no conflicts of interest.

\section{References}

1. Nakai S, Suzuki K, Masakane I, Wada A, Itami N, et al. (2010) Overview of regular dialysis treatment in Japan (as of 31 December 2008). Ther Apher Dial 14: $505-540$

2. Foley RN (2004) Comparing the incomparable: hemodialysis versus peritoneal dialysis in observational studies. Perit Dial Int 24: 217-221.
3. Jaar BG, Coresh J, Plantinga LC, Fink NE, Klag MJ, et al. (2005) Comparing the risk for death with peritoneal dialysis and hemodialysis in a national cohor of patients with chronic kidney disease. Ann Intern Med 143: 174-183.

4. Tam $P$ (2009) Peritoneal dialysis and preservation of residual renal function Perit Dial Int 29 Suppl 2: S108-110.

5. Vilar E, Farrington K (2011) Emerging importance of residual renal function in end-stage renal failure. Semin Dial 24: 487-494.

6. Brener ZZ, Thijssen S, Kotanko P, Kuhlmann MK, Bergman M, et al. (2011) The impact of residual renal function on hospitalization and mortality in incident hemodialysis patients. Blood Purif 31: 243-251.

7. Diaz-Buxo JA, Gotch FA, Folden TI, Rosenblum S, Zazra J, et al. (1999) Peritoneal dialysis adequacy: a model to assess feasibility with various modalities. Kidney Int 55: 2493-2501.

8. Agrawal B, Wolf K, Berger A, Luft FC (1996) Effect of antihypertensive treatment on qualitative estimates of microalbuminuria. J Hum Hypertens 10: 551-555.

9. Fukui H, Hara S, Hashimoto Y, Horiuchi T, Ikezoe M, et al. (2004) Review of combination of peritoneal dialysis and hemodialysis as a modality of treatment for end-stage renal disease. Ther Apher Dial 8: 56-61.

10. Hoshi H, Nakamoto H, Kanno Y, Takane H, Ikeda N, et al. (2006) Long-term follow-up of patients treated with a combination of continuous ambulatory peritoneal dialysis and hemodialysis. Adv Perit Dial 22: 136-140.

11. Kawanishi H, Hashimoto $Y$, Nakamoto H, Nakayama M, Tranaeus A (2006) Combination therapy with peritoneal dialysis and hemodialysis. Perit Dial Int 26: 150-154.

12. Kawanishi H, McIntyre C (2008) Complementary use of peritoneal and hemodialysis: therapeutic synergies in the treatment of end-stage renal failure patients. Kidney Int Suppl: S63-67.

13. Kawanishi $\mathrm{H}$, Moriishi $\mathrm{M}$ (2007) Clinical effects of combined therapy with peritoneal dialysis and hemodialysis. Perit Dial Int 27 Suppl 2: S126-129.

14. Kawanishi H, Moriishi M, Tsuchiya S (2002) Five years' experience of combination therapy: peritoneal dialysis with hemodialysis. Adv Perit Dial 18 $62-67$

15. Van Biesen W, Vanholder RC, Veys N, Dhondt A, Lameire NH (2000) An evaluation of an integrative care approach for end-stage renal disease patients. J Am Soc Nephrol 11: 116-125.

16. Uchida K, Shoda J, Sugahara S, Ikeda N, Kobayashi K, et al. (2007) Comparison and survival of patients receiving hemodialysis and peritoneal dialysis in a single center. Adv Perit Dial 23: 144-149.

17. Morton RL, Devitt J, Howard K, Anderson K, Snelling P, et al. (2010) Patient views about treatment of stage $5 \mathrm{CKD}$ : a qualitative analysis of semistructured interviews. Am J Kidney Dis 55: 431-440.

18. Shinzato T, Nakai S, Akiba T, Yamagami S, Yamazaki C, et al. (1999) Report of the annual statistical survey of the Japanese Society for Dialysis Therapy in 1996. Kidney Int 55: 700-712.

19. Han SH, Lee SC, Ahn SV, Lee JE, Choi HY, et al. (2007) Improving outcome of CAPD: twenty-five years' experience in a single Korean center. Perit Dial Int 27: $432-440$. 